\title{
Relationship between Vortex Ring in Tail Fin Wake and Propulsive Force
}

\author{
Naoto Imamura, Kazuo Matsuuchi \\ Graduate School of Systems and Information Engineering, University of Tsukuba, \\ Tennoudai 1-1-1, Tsukuba, Ibaraki 305-8573, Japan \\ imamuran@cem.casio.co.jp
}

\begin{abstract}
Our aim was to investigate the three-dimensional (3D) vortex ring in the wake of a tail fin and to clarify the propulsion mechanism of dolphins and fish. In this study, we replaced a tail fin in pitching motion with an oscillating wing having a drive unit. The flow fields around the wing were measured by stereoscopic particle image velocimetry. To visualize the 3D structure of the vortex in the wake, we determined the flow fields in equally spaced cross-sectional planes. We reconstructed the $3 \mathrm{D}$ velocity fields from the velocity data with three components in two dimensions. We visualized the 3D vortex structure from these velocity data and plotted an isovorticity surface. As a result, we found that the vortex ring was generated by the kick-down and kick-up motions of the wing and that the wake structure was comparable with that obtained numerically. Moreover, we calculated the propulsive forces from the temporal variations in circulation and in the area surrounded by the vortex ring.
\end{abstract}

\section{Introduction}

Aquatic animals swimming at high speeds in water, such as dolphins, achieve high propulsive efficiency. However, the hydrodynamic mechanism of dolphins is not clear because an analytical approach to large-scale wing oscillation has not yet been established. It is difficult to analyze the flow field around these wings because of the complexity resulting from its three-dimensionality and unsteadiness.

Many experiments and numerical calculations have been conducted to clarify the relationship between the flow field around a swimming fish and its propulsive force (see Triantafyllou et al., 2004 for a review of studies published before 2004). In earlier studies, the main concern was to gain insight into the twodimensional (2D) flow of an oscillating wing using numerical simulations and experiments (see Anderson et al., 1998). A highly important discovery was that 
unsteady mechanisms play a very important role. High values of the lift coefficient were found to be associated with the formation of a leading-edge vortex with a large scale per half-cycle, resulting eventually in the formation of a reverse Karman street. The appearance of the vortex street was found to be strongly related to a nondimensional parameter called the Strouhal number. Studies of oscillating wings made of rigid material were extended to flexible wings (Alben, 2009), and the conditions that yielded the maximum power output or high efficiency were also discussed. The importance of such large-scale vortices was also recognized in relation to a dynamic stall or high-lift mechanism in an accelerated flow or to translating bodies such as an aerofoil with a large angle of attack (Izumi and Kuwahara., 1983; Morikawa and Grönig, 1995; Soria et al., 2003; Troolin et al., 2006; Sengupta et al., 2007).

Müller et al. (1997) analyzed the wake behind a continuously swimming mullet by using particle image velocimetry (PIV); they found that undulatory oscillation of the body generated less than half of the total energy, and the remainder was generated by the tail. Drucker and Lauder (1999) reconstructed the wake structure of the pectoral fin of a bluegill sunfish based on PIV data and also estimated the thrust and lift due to the fin. Nauen and Lauder (2002) measured the flow field around the tail fin of a swimming mackerel from various directions using PIV and predicted the structure of the continuous vortex rings formed in the fin wake from the measured velocity data in each plane. They also estimated the propulsive force by postulating vortex ring formation. Wolfgang et al. (1999) performed threedimensional (3D) numerical calculations using a computational model based on a fish body shape together with a fin; they concluded that for the flow field around the fish, a 2D approximation is possible in the horizontal plane, but the threedimensionality is high in the vertical plane and was highest near the fin tip. They solved the interaction of the body-generated vorticity with a strong wake shed from the tail fin by assuming the flow to be irrotational, with the exception of thin wakes.

In addition to studies of real fish, model experiments or numerical simulations have been conducted, focusing on the unsteady dynamics produced by an oscillating wing with a finite span. A straightforward and simpler method of 
observing the 3D structure of the wake is to use streak lines visualized using dye (von Ellenrieder et al., 2003; Parker et al., 2005; Buchholz and Smits, 2006; Buchholz and Smits, 2008). Very complex structures of the unsteady and 3D characteristics were proposed as a three-dimensional vortex skeleton. However, many researchers (Hama, 1962; Coutanceau and Defaye, 1991; von Ellenrieder et al., 2003) noted the limitations of this method in applications to unsteady flows. To test the interpretation of the wake structure found by dye-flow observations, many of these authors conducted planar measurements using PIV (Parker et al., 2005; Buchholz and Smits, 2006; Parker et al., 2007b; Buchholz and Smits, 2008).

In addition to experimental studies, numerical analysis is becoming a powerful tool for analyzing the wake structure behind or around a 3D oscillating wing (Blondeaux et al., 2005; Dong et al., 2006). These researchers visualized the wake structure by drawing the contour of the vorticities in detail. However, no 3D results have been obtained experimentally for comparison with the structure constructed numerically.

Using a quite different approach, Gharib and others proposed a new idea that would lead to an optimal condition for vortex ring generation based on a pistonnozzle model; they discussed the condition by introducing a new parameter, the formation number (Gharib et al., 1998; Dabiri and Gharib, 2005; Linden and Turner, 2004). Although the kinematics of the original model differs from that of generation by an oscillating wing, this concept may illuminate the optimal condition for high-efficiency vortex ring generation in an oscillating wing.

These studies improved our understanding of the tail fin propulsion mechanism. However, either the flow fields were predicted from only 2D velocity data or there was no experimental evidence indicating their 3D structure. Therefore, our aim was to visualize the $3 \mathrm{D}$ vortex structure in the wake of a tail fin and to investigate the relationship between vortex ring formation and the propulsive force. We conducted 3D measurements using the stereoscopic particle image velocimetry (stereo-PIV) (Prasad and Adrian, 1993) of an oscillating wing with a drive unit. 
We visualized the vortex structure using experimental data and calculated the propulsive force from the circulation and area of the vortex ring.

\section{Methods}

Experiments using live animals encounter severe problems with individual differences and repeatability. Therefore, we replaced a tail fin in a pitching motion with an oscillating wing having a drive unit. The unit has a function of transforming from rotational motion to oscillation via a crank mechanism. A schematic of the experimental setup is shown in Fig. 1. Experiments were conducted in a water tunnel having a length of $4.6 \mathrm{~m}$, a span of $2.0 \mathrm{~m}$, and a depth of $1.2 \mathrm{~m}$, with a glass-windowed observation section. The water temperature was set to $27^{\circ} \mathrm{C}$ (density $\rho=0.997 \mathrm{~g} / \mathrm{cm}^{3}$ ). The flow field was observed and illuminated through glass windows on both sides of the water tunnel. The pitching angle and frequency of the wing drive unit could be arbitrarily adjusted. In this experiment, $x$ denotes the direction of flow, $y$ denotes the span direction of the tunnel, and $z$ denotes the height.

The flow in the water tank was visualized using seeded nylon particles (DaicelEvonik, DAIAMID, Polyamide12, diameter of $100 \mu \mathrm{m}$, specific gravity of 1.03). The responses of the particles in an oscillating flow were confirmed to be reliable in a $1 \mathrm{kHz}$ frequency range. A laser sheet $8 \mathrm{~mm}$ in thickness produced by a double-pulsed Nd:YAG laser (New Wave Research, Solo 120XT, 15 Hz) irradiated the $y-z$ plane from the side of the tunnel. Two CCD cameras (Kodak, ES1.0, 1008 pixels $\times 1024$ pixels, $30 \mathrm{fps}$ ), referred to as CCD cameras 1 and 2 in Fig. 1, were placed on either side of the tunnel to capture images of the particles. The angle between the optical axes of the two cameras was set to about $90^{\circ}$, and lenses were mounted so as to satisfy the Scheimpflug condition (Prasad and Jensen, 1995). Two prism containers made of acrylic and filled with water were placed outside the tunnel and used to make the optical axis perpendicular to the air-tank interface.

Data capture was controlled by a rotary encoder (Ono Sokki, LG-916) and a pulse generator (Quantum Composers, Inc., 9314). The encoder detected the rotational 
position of the rotating disk in the drive unit and output the digital signal by dividing it into 24 segments at equal time intervals for a pitching motion cycle. The output signal was forwarded to the pulse generator. A trigger signal was then sent from the output of the pulse generator to the laser oscillator and the CCD cameras. The images captured by these CCD cameras were stored in the host memory of a PC via image grabbers (Coreco Imaging, Inc., PC-DIG).

We used a stereoscopic PIV system built in-house that includes data acquisition and a method of determining the velocity fields. The data acquisition and analysis methods are described in detail in Sakakibara et al. (2004) together with the error estimation. The stereo-PIV measurement region was set to about $600 \mathrm{~mm} \times 500$ $\mathrm{mm}$ in the $y$-z plane. All the three components of velocity were determined completely at points spaced at $10 \mathrm{~mm}(=\Delta y=\Delta z)$ in the plane. To capture the 3D vortex structure in the wake, we measured the flow fields of many equally spaced cross-sections. We changed the measurement plane by moving the system of the oscillating wing in the direction normal to the laser sheet, instead of moving the optical system, because it was difficult to position the system exactly. The measurement planes are shown in Fig. 2. Thirty-one cross-sections were measured by moving the wing system up to $600 \mathrm{~mm}$ in $20 \mathrm{~mm}(=\Delta x)$ steps in the upstream direction (negative $x$ direction). The movement was carried out manually, in a careful manner. The positioning accuracy was less than $0.5 \mathrm{~mm}$. This value is considerably small compared to the position uncertainty of the cross-correlation method. Among the planes, the plane $100 \mathrm{~mm}$ from the tips of the wing was chosen as a basis. Then, 480 image pairs (20 image pairs $\times 24$ phases) were taken at each cross-section. The velocities were determined in each $y$-z plane using two particle images taken at two instants at an interval of $2.5 \mathrm{~ms}$. Moreover, unsynchronized fluctuations were removed by phase-averaging over 20 pitching cycles.

The physical coordinates and the corresponding coordinates on the image plane were calibrated as follows. A calibration plate consisting of a $10 \times 10 \mathrm{~mm}^{2}$ lattice was used to completely calibrate the $y$-z coordinates of the lattice points. Other points were interpolated using polynomials. For calibration in the $x$ direction, the same lattice was used on the plane $10 \mathrm{~mm}$ away from the first one. The movement 
accuracy of the lattice plate was achieved by a programmable stepping motor. Our measurement system was the same as that described in Sakakibara et al. (2004), and research conducted with the same system, including a large facility such as the water tunnel, has already been published (Matsuuchi et al., 2009). We followed the same procedure with no modifications. The corresponding uncertainty of the velocity measurement was estimated by assuming the subpixel accuracy of the particle displacement measurement to be 0.1 pixels. The estimated uncertainty was $26 \mathrm{~mm} / \mathrm{s}$ for the $w$ component and $39 \mathrm{~mm} / \mathrm{s}$ for both the $u$ and $v$ components. The errors were $5.2 \%$ and $7.8 \%$, respectively, relative to a uniform velocity of $500 \mathrm{~mm} / \mathrm{s}$. The vorticity was estimated by substituting the space derivatives of the velocities with their central difference at intervals of $20 \mathrm{~mm}$ (= $\Delta x)$ in the $x$ direction and $10 \mathrm{~mm}(=\Delta y=\Delta z)$ in the two remaining directions. The central difference had second-order accuracy. The finite-difference approximation error is estimated to be at most on the order of $10^{-3}$, which is negligibly small compared with the error in the cross-correlation method.

The shape and material of the wing set up in the unit are shown in Fig. 3. The wing was made of laminated carbon-fiber-reinforced plastic (CFRP), and its design was based on the form of a $1.2 \mathrm{~m}$ long fish swimming with carangiform motion (Breder, 1926). To improve the elasticity, the thickness of the wing was varied by using a laminated construction that became thinner from the root toward the tip. The laminated form has been continually revised for over ten years at the Musashi Institute of Technology in Japan. We studied this laminated wing and estimated the flexibility of the wing, whose design was propriety, by measuring the tip displacement of the wing. As shown in Fig. 3, the stepwise change in thickness is $0.3 \mathrm{~mm}$ at maximum. According to the laminar boundary theory, the thickness at $x=80 \mathrm{~mm}$ is about $2 \mathrm{~mm}$. The steps were completely within the boundary layer. Accordingly, the effect of discrete changes may not greatly affect the total flow field.

A schematic of the pitching motion of the wing and its drive unit is shown in Fig. 4. In the holding part of the wing, there is an asymmetry between the upper and lower sides. This asymmetry may affect the structure of wakes in the kick-up and kick-down. The pitching angle $\theta_{\mathrm{p}}$ measured from the horizontal plane $(z=0)$ was 
positive for counterclockwise rotation and negative for clockwise rotation. Moreover, the pitching angles of the upper and lower dead points are designated as $\theta_{\mathrm{a}}$ and - $\theta_{\mathrm{a}}$, respectively. The distance between the upper and lower dead points at the wing tip in pitching motion was denoted as the double amplitude $a$ (see Fig. $4)$.

The oscillating wing was set at a depth of $500 \mathrm{~mm}$ below the surface. The Froude number was 0.226 on the basis of a uniform velocity and depth. The effect of the free surface on the flow was estimated and was confirmed to be negligible. The experimental conditions were selected according to those of a live animal. The Strouhal number $S t$ is defined here as

$$
S t=\frac{f_{\mathrm{p}} a}{U_{0}},
$$

where $f_{\mathrm{p}}$ is the pitching frequency, and $U_{0}$ is the flow velocity of the water tunnel. Taylor et al. (2003) estimated the Strouhal number for fish and found that it ranged from 0.2 to 0.4 , irrespective of the size and swimming method. Therefore, experiments were conducted under the following conditions: $U_{0}=0.5 \mathrm{~m} / \mathrm{s}, f_{\mathrm{p}}=1.0$ $\mathrm{Hz}$, and $\theta_{\mathrm{a}}=15^{\circ}(a=0.195 \mathrm{~m})$, with $S t=0.39$. The Reynolds number was estimated to be $1.6 \times 10^{5}$ for water at $27{ }^{\circ} \mathrm{C}$ using the chord length as a characteristic length and the uniform velocity as a characteristic velocity. Furthermore, to investigate the relationships between the variation in propulsive force and the flow field, we conducted further measurements with $\theta_{\mathrm{a}}=20^{\circ}$ and $25^{\circ}$. The experimental conditions are summarized in Table 1.

To determine the fin flexibility quantitatively, we measured deflections of the fin tip in the period immediately after the transition from kick-up to kick-down. The measured deflection at the tip was $51.2 \mathrm{~mm}$ for the pitching angle $\theta_{\mathrm{a}}=15^{\circ}$. The corresponding value for $\theta_{\mathrm{a}}=25^{\circ}$ was calculated as $71.6 \mathrm{~mm}$..

\section{Visualization of the 3D vortex structure}

Temporal variations in the pitching motion were investigated as a function of the dimensionless time, determined by the product of the time and the pitching 
frequency, $t^{*}\left(=t \cdot f_{\mathrm{p}}\right)$. The upper dead point was assumed to be $t^{*}=0$, and the lower dead point was assumed to be about $t^{*}=0.5$.

We reconstructed the 3D velocity fields from the velocity data with three components in two dimensions obtained by stereo-PIV. Three components of the vorticity, $\omega_{x}, \omega_{y}$, and $\omega_{z}$, and the absolute value $|\omega|$, defined as

$$
|\omega|=\sqrt{\omega_{x}^{2}+\omega_{y}^{2}+\omega_{z}^{2}},
$$

were calculated from the reconstructed velocity data. As noted by Adrian et al. (2000), a shear layer would make the vortex shape unclear. A quick way of avoiding this difficulty is to use an isosurface of the swirling strength instead of that of the vorticity strength. However, because the vortex is not weak enough to be cleared away by the shear layer, as numerical results have clearly indicated (see Blondeaux et al., 2005; Dong et al., 2006), we selected the isosurface of the vorticity to visualize the 3D vortex structure. The vortex structures created by kick-down and kick-up for $\theta_{\mathrm{a}}=15^{\circ}$ at $t^{*}=0.45$ and 0.95 are shown in Figs. 5 (a)(c) (an animation is provided in Online Resource 1). In these figures, we show the isosurface of the vorticity for $|\omega|=10 \mathrm{~s}^{-1}$, (a) the isosurface in $x-y-z$ space, (b) the isosurface and streamline in the central plane of the wing (in the $x-z$ plane, $y=0$ ) viewed from the side, and (c) the isosurface and streamline in the horizontal plane (in the $x-y$ plane, $z=0$ ) viewed from the top. The left-hand panel of Fig. 5 shows the vortex structure just before the wing reaches the lower dead point by kickdown at $t^{*}=0.45$. In the process leading up to this time, the vortex ring generated by kick-up in the preceding cycle is shed, and a new vortex ring is formed by kick-down in the current cycle. Conversely, the right-hand panel of Fig. 5 shows the vortex structure just before the wing reaches the upper dead point, and a new vortex ring is formed by kick-up at $t^{*}=0.95$. These figures also show that these vortex rings connected with each other in the back and front. In Figs. 5 (b) and (c), it is clear that a type of jet flow passed through in the vortex ring generated by the wing motion. Hence, it was thought that a zigzagging jet flow, which passed sequentially through two types of vortex rings, occurred in the pitching motion. A slight asymmetry between the kick-up and kick-down motions can particularly be observed in Fig. 5 (b). The asymmetry may have resulted from the motion of the upstream part of the wing including the holding part (see Fig. 4). 
The result for the three components of the vorticity, $\omega_{x}, \omega_{y}$, and $\omega_{z}$, extracted from the 3D vortex structure of Fig. 5 are shown in Figs. 6 (a)-(c), respectively (an animation is provided in Online Resource 2). These figures show the isosurfaces of the vorticity for $\pm 10 \mathrm{~s}^{-1}$; positive vorticity appears in red, and negative vorticity appears in blue. In these figures, $\theta_{\mathrm{a}}=15^{\circ}$ at $t^{*}=0.45$ and 0.95 , as shown in Fig. 5 .

From Fig. 6 (a) for $\omega_{x}$, it is clear that a pair of trailing vortices with positive and negative signs was released by kick-down and kick-up. From Fig. 6 (b), it is clear that $\omega_{y}$ was highly 2D over the wingspan. In this figure, the vorticity component $\omega_{y}$ due to wing motion took negative and positive values in kick-down and kickup, respectively. Such a series of vortices $\omega_{\mathrm{y}}$ with positive and negative signs is known to form a reverse Karman vortex street (Anderson et al., 1998; Triantafyllou et al. 2000). In comparison to the other rotational components, the vorticity component $\omega_{z}$ was scarcely discernible near the wing [Fig. 6 (c)].

We confirmed that the reverse Karman vortex street $\left(\omega_{y}\right)$ is connected with the trailing vortex $\left(\omega_{x}\right)$, and the wing wake as a whole forms a vortex ring, as shown in Fig. 5. We visualized the vortex structure in the wing wake on the basis of the experimental data obtained by stereo-PIV, although so far the wake structure has been deduced from the results of 2D PIV.

Geometric similarities were found between our reconstructed vortex structure and the numerical results of Blondeaux et al. (2005) and Dong et al. (2006). Dye flow visualization with streak lines also shows that the wake vorticity is dominated by a single pair of intense counter-rotating vortices. However, each member of the vortex pair consists of two smaller co-rotating vortices (Parker et al., 2007a). In addition, the vortex structure obtained by dye-flow visualization (Buchholz and Smits, 2006; Buchholz and Smits, 2008) is more complex than that we obtained by our PIV measurement. For example, two smaller co-rotating vortices (Parker et al., 2007a) were not discerned in the wake just behind the wing. Dye flow visualization is a useful means of obtaining an overall picture of the 3D flow structure. However, many researchers have noted the limitations of this method in applications to unsteady flows and the possibilities for misunderstanding the 
unsteady characteristics (Hama, 1962; Coutanceau and Defaye, 1991; von Ellenrieder et al., 2003).

\section{Propulsive force based on vortex ring}

\subsection{Estimation of propulsive force}

To clarify the relationship between the vortex ring in the wing wake and the propulsive force in wing motion, we calculated the force using the temporal variations in the momentum of a vortex ring (Milne-Thomson, 1966). The momentum is derived from the "impulse" (Lamb, 1932) and in this case has the form

$$
M=\rho \Gamma A,
$$

where $\Gamma$ is the circulation along a closed loop of a vortex filament of the vortex ring, $A$ is the area surrounded by a vortex ring, and $\rho$ is the density of the fluid. A similar approach has been used by many authors (for example, Dickinson, 1996; Drucker and Lauder, 1999; Sane, 2003). The force $F$ resulting from the formation of the vortex ring is obtained by differentiating Eq. 3 with respect to time according to Newton's second law of motion:

$$
F=\rho \frac{\mathrm{d}}{\mathrm{d} t}(\Gamma A) .
$$

Eq. 4 can be rewritten by taking into account the temporal changes in both the ring circulation and ring area (Dickinson, 1996):

$$
F=\rho\left(\Gamma \frac{\mathrm{d} A}{\mathrm{~d} t}+A \frac{\mathrm{d} \Gamma}{\mathrm{d} t}\right)
$$

Here, considering only the component of the propulsive force, from Eq. 5, we obtain

$$
F_{x}=\rho\left(\Gamma \frac{\mathrm{d} A_{y z}}{\mathrm{~d} t}+A_{y z} \frac{\mathrm{d} \Gamma}{\mathrm{d} t}\right)
$$

where $A_{y z}$ is the area projected in the $y-z$ plane of area $A$. To determine $F_{x}$ from Eq. 6, the temporal changes in $\Gamma$ and $A_{y z}$ have to be calculated from the development of a vortex. 
Fig. 7 illustrates a method for calculating the circulation $\Gamma$. The circulation was estimated by choosing, as a typical value, the vorticity $\omega_{y}$ in the central plane of the wing, i.e., in the $x-z$ plane $(y=0)$. We integrated over the area of $\omega_{y} \leq 0$ in kick-down and over that of $\omega_{y} \geq 0$ in kick-up because $\omega_{y}$ has a negative value in kick-down and a positive value in kick-up, as shown in Fig. 6 (b). Note that the vortex in the previous pitching cycle was not included in the integration. The integration range in the $x$ direction was limited to $0 \leq x \leq x_{\max }+0.2 \mathrm{~m}$, where $x_{\max }$ is the $x$ coordinate at which $\omega_{y}$ peaks in the plane. In the left and right panels of Fig. 7, the two integration ranges are identified by blue and red frames, respectively.

Fig. 8 shows a method for calculating the area $A_{y z}$. The area was calculated first by finding the points lying on a vortex filament and then by defining the polygon connecting these points on the filament. First, we defined an angle $\theta_{\mathrm{w}}$ that measured the direction viewed from the midpoint of the line connecting the two wing tips, as shown in Fig. 8 (a). Next, the point giving the peak of $|\omega|$ in the plane $\theta_{\mathrm{w}}=0^{\circ}$ was found. If the plane differed from the lattice position of the experimental data, a linear interpolation of $|\omega|$ was made. A similar procedure was then carried out by moving $\theta_{\mathrm{w}}$ by $10^{\circ}$ increments in the range $10^{\circ} \leq \theta_{\mathrm{w}} \leq 180^{\circ}$. In Fig. 8 (a), the detected points are depicted by red circles. Finally, we determined the vortex ring area by connecting the detected points, as shown in Fig. 8 (b), and calculated $A_{y z}$ by projecting the area to the $y-z$ plane, as shown in Fig. 8 (c). Note that the plane surrounded by a vortex ring exchanges inside out sequentially in kick-down and kick-up. The sign of the area $A_{y z}$ was defined as negative in kick-down and positive in kick-up.

\subsection{Calculated circulation and area of the vortex ring, and propulsive force}

The calculation results for the circulation $\Gamma$ are shown in Fig. 9. The horizontal axis represents the dimensionless time $t^{*}$, and the vertical axis denotes the circulation. The wing acceleration (at an arbitrary scale) is also drawn for convenient interpretation. The magnitude of $\Gamma$ increases with time and then 
becomes constant near $t^{*}=0.3$ and 0.8 , where the wing lies almost in the horizontal plane. Therefore, we speculate that $\Gamma$ increases in the time period when the wings are accelerating and then becomes constant in the time period when the wings are decelerating in a pitching motion. In Fig. 9, the temporal variations in the wing acceleration,

$$
\alpha_{p}=\frac{d}{d t}\left|\frac{d \theta_{p}}{d t}\right|,
$$

were drawn at an arbitrary scale for convenience. This means that the wing deceleration did not contribute to vortex generation. The visualization shows that vortices were shed at the instant the wing was near the upper or lower dead point. The vortices form a reverse Karman vortex street. In this respect, the video (Online Resource 2) is helpful for understanding the situation. Moreover, because the increase in $\Gamma$ becomes more remarkable as the pitching amplitude $\theta_{\mathrm{a}}$ is increased, it is probable that the increase in $\Gamma$ becomes more remarkable as the wings accelerate.

The time variations in the area $A_{y z}$ are shown in Fig. 10. The horizontal axis represents the dimensionless time $t^{*}$, and the vertical axis denotes $A_{y z}$. No data were plotted during the transient period of the wing from kick-up to kick-down and vice versa because of the difficulty in determining the area. In this figure, $A_{y z}$ begins to increase about 0.1 later than the instant at which the direction of the wing motion changes. The increase rate of $A_{y z}$ is large when the pitching amplitude $\theta_{\mathrm{a}}$ is large. It is plausible that as the wing moves further, the trailing vortex leaving both edges of the wing becomes longer, so a long vortex ring is formed.

Substituting the circulation $\Gamma$ and the area $A_{y z}$ in Eq. 6, we can calculate the propulsive force $F_{x}$, as shown in Fig. 11. The horizontal axis measures the dimensionless time $t^{*}$, and the vertical axis denotes $F_{X}$. The latter has two peaks in a pitching cycle; they occur at the instants $t^{*}=0.3$ and 0.8 , which is just when the wing is almost in the horizontal plane. The reason is that the speed of the wing is highest at these instants. Moreover, $F_{X}$ becomes larger when pitching amplitude $\theta_{\mathrm{a}}$ is larger. The force variations are different from that calculated by Parker et al. (2007a), who showed that the peaks in the force appear at the instants $t^{*}=3 / 8$ 
and $t^{*}=7 / 8$ using a formula commonly used for steady flows. It is not clear whether the difference arises from the use of the formula or from differences due to the kinematic conditions.

Note that the calculated $F_{X}$ could have a larger value than the actual propulsive force measured separately. The cause may lie in the determination of the circulation $\Gamma$. In this study, $\Gamma$ was evaluated as a typical value of the vorticity $\omega_{y}$ in the central plane of the wing, i.e., in the $x-z$ plane at $y=0$. However, it is doubtful that $\Gamma$ is constant along the circumference of the vortex ring. It is probable that the circulation calculated in the central plane is largest on the circumference of the vortex ring. Therefore, the circulation at other positions on the circumference of the vortex ring will be discussed in the next section.

\section{Discussion}

First, we discuss the 3D effect on a vortex ring. In many investigations reported to date, 3D flow structures have been reconstructed using 2D data obtained in one plane under a certain condition. We attempted to check the validity of this method by reconstructing the 3D vortex structure in the wing wake using 2D PIV data obtained in the $y-z$ plane. We arranged time series data in the hypothetical $x$ direction (the flow direction) using Taylor's frozen-flow hypothesis (Taylor, 1938) and obtained 3D spatial data. To reconstruct the 3D wake structure from the 2D data, we introduce the hypothetical coordinate $x^{*}$, defined as

$$
x^{*}=U_{c} \cdot t \text {, }
$$

where $U_{\mathrm{c}}$ is the convection velocity estimated by averaging the axial velocity $u$ spatially and temporally.

The 3D vortex structure thus obtained is shown in Fig. 12 (an animation is provided in Online Resource 3). The vortex structure was built with the data in the $y-z$ plane at $x=0.3 \mathrm{~m}$ from the wing tip. The isosurface of the vorticity for $|\omega|=$ $10 \mathrm{~s}^{-1}$ is depicted for $\theta_{\mathrm{a}}=15^{\circ}$ at $t^{*}=0.95$, as in the right-hand panel in Fig. 5. There are clearly two differences between Figs. 12 and. 5. The first is that the vortex spreading in the $z$ direction seen in Fig. 5 (b) is not as large as that in Fig. 
12 (b). The second is that the shrinking of the vortices into the centerline seen in Fig. 5 (c) is scarcely detectable in Fig. 12 (c). The vortex ring seems to flow downstream while maintaining a constant diameter. Therefore, it is concluded that the vortex structure in the real wing wake cannot flow downstream with a fixed shape once generated; thus, it seems to be difficult to correctly determine its structure using only 2D data obtained in a fixed plane.

In the previous section, the circulation $\Gamma$ was determined only in the central plane. To evaluate the momentum of a vortex ring in the wing wake in more detail, the circulation around the vortex rings at points other than the point in the central plane should be calculated. Fig. 13 illustrates how to calculate this circulation. Here, only the circulation near the middle of the trailing vortices was calculated as an example. All the variations along a ring were impossible to calculate because the vortices overlapped.

We first define a unit tangent $\boldsymbol{t}$. On the vortex filament, we consider two position vectors close to each other, $\boldsymbol{s}$ and $\boldsymbol{s}+\mathrm{d} \boldsymbol{s}$. The unit vector is then defined by $\boldsymbol{t}=$ $\mathrm{d} \boldsymbol{s} / \mathrm{d} s(\mathrm{~d} s=|\mathrm{d} \boldsymbol{s}|)$. The vector is expressed as $\left(\sin \theta_{t} \cos \phi_{t}, \sin \theta_{t} \sin \phi_{t}, \cos \theta_{t}\right)$, where $\phi_{t}$ and $\theta_{t}$ are the azimuthal and polar angles, respectively [see Fig. 13 (a)]. Two tangent vectors corresponding to $\theta_{\mathrm{w}}=20^{\circ}$ (left-hand side of the wing, $\mathrm{LH}$ ) and $160^{\circ}$ (right-hand side of the wing, $\mathrm{RH}$ ) on the vortex filament are shown in Fig. 13 (a). Next, we consider two planes perpendicular to the tangent vectors that pass through the points $\theta_{\mathrm{w}}=20^{\circ}$ and $160^{\circ}$, as shown in Fig. 13 (b). In calculating the circulation on both sides of the wing, the integration is performed in the left and right planes separately. The component of the vorticity $\omega=\left(\omega_{x}, \omega_{y}, \omega_{z}\right)$ normal to the plane, $\omega^{*}$, is calculated as $\omega^{*}=\omega \cdot \boldsymbol{t}$. Finally, the circulation $\Gamma$ was obtained by integrating $\omega^{*}$ in each plane, as shown in Fig 13 (c). Note that $\omega^{*} \leq 0$ in kickdown, and $\omega^{*} \geq 0$ in kick-up, as shown in Fig. 7 .

The results of the circulation $\Gamma$ are summarized in Table 2 . They were estimated at the same instants as those shown in Fig. 5 . Table 2 shows that the circulation $\Gamma$ in the left-hand and right-hand planes was about 54\% smaller in kick-down and 66\% smaller in kick-up than those in the central plane. Therefore, if we assume that the value increases at a fixed rate from the wing tip toward the central plane of the 
wing, the propulsive force $F_{X}$ shown in Fig. 11 would be overestimated by about $30 \%$ in kick-down and 20\% in kick-up motion.

Last we mention about the formation time proposed by Gharib et al. (1998). According to Linden et al. (2004), the formation time corresponds to the inverse Strouhal number,

$$
\frac{U_{0}}{f_{p} a}=\frac{T U_{0}}{a}
$$

This is a formal adaption of plug aspect ratio. Note that the vortex rings are oblique to the main flow and the generation of them occupies a half of the entire oscillating period $T$, it is plausible to replace the double amplitude $a$ with $a \sin \left(\alpha_{r}\right)$ and $T$ with $T / 2$, where $\alpha_{r}\left(\cong 32^{\circ}\right)$ is an angle of attack of the slanted vortex ring. Hence we have the formation time of 2.4 for our oscillating wing. This value may be comparable to that for an optimal formation condition of a vortex ring.

\section{Conclusions}

We aimed to clarify the relationship between the flow field around a tail fin and the propulsive force by conducting 3D measurements of the wing wake in a water tunnel using stereo-PIV and an oscillating wing with a drive unit. We then visualized and determined the 3D structure of vortex ring formation in the wing wake on the basis of the experimental data. We demonstrated that the wake consists of a series of a vortex pairs of intense counter-rotating vortex rings, and each vortex ring consists of a trailing vortex and reverse Karman vortex. The reconstructed structure of the wake is very close to that obtained in numerical simulations. We also showed that 3D structures based on 2D data do not describe the vortex characteristics correctly. Moreover, we calculated the propulsive forces arising from vortex ring formation. We then found that the circulation $\Gamma$ in kickdown first increases as a function of time and then becomes constant after about $t^{*}$ $=0.3$, when the wing is almost in the horizontal plane. The circulation in kick-up also increases first and becomes constant after about $t^{*}=0.8$. It also followed that the rate of increase in $A_{y z}$ is large for a large pitching amplitude $\theta_{\mathrm{a}}$. The propulsive 
forces resulting from vortex ring formation were estimated and demonstrated two peaks in each pitching cycle, which is inherent in the formation of a vortex ring.

\section{Acknowledgments}

This study was supported by a Grant-in-Aid for Challenging Exploratory Research (23650383) from the Japan Society for the Promotion of Science. The authors thank Professor Jun Sakakibara of the University of Tsukuba for providing many useful comments and the technical support for using the PIV measurement system. The authors also thank the reviewers for insightful comments and the editor for helpful guidance.

\section{References}

Adrian RJ, Christensen KT, Liu Z-C (2000) Analysis and interpretation of instantaneous turbulent velocity fields. Exp. Fluids 29: 275-290

Alben S (2009) Simulating the dynamics of flexible bodies and vortex sheets. J. Comput. Phys.

228: 2587-2603

Anderson JM, Streitlien K, Barrett DS, Triantafyllou MS (1998) Oscillating foils of high propulsive efficiency. J. Fluid Mech. 360: 41-72

Blondeaux P, Formarelli F, Guglielmini L, Triantafyllou MS, Verzicco R (2005) Numerical experiments on flapping foils mimicking fish-like locomotion. Phys. Fluids 17(113601) Breder CM (1926) The locomotion of fishes. Zoologica 4: 159-297

Buchholz JHJ, Smits AJ (2006) On the evolution of the wake structure produced by a low-aspectratio pitching panel. J. Fluid Mech. 546: 433-443

Buchholz JHJ, Smits AJ (2008) The wake structure and thrust performance of a rigid low-aspectratio pitching panel. J. Fluid Mech. 603: 331-365

Coutanceau M, Defaye AY (1991) Circular cylinder wake configurations: A flow visualization survey. App. Mech. Rev. 44: 255-305

Dabiri JO, Gharib M (2005) The role of optimal vortex formation in biological fluid transport. Proc. Roy. Soc. B272: 1557-1560

Dickinson MH (1996) Unsteady mechanisms of force generation in aquatic and aerial locomotion. Am. Zool. 36: 537-554

Dong H, Mittal R, Najjar FM (2006) Wake topology and hydrodynamic performance of lowaspect-ratio flapping foils. J. Fluid Mech. 566: 309-343

Drucker EG, Lauder GV (1999) Locomotor forces on a swimming fish: Three-dimensional vortex wake dynamics quantified using digital particle image velocimetry. J. Exp. Biol. 202: 2393-2412 Gharib M, Rambod E, Shariff K (1998) A universal time scale for vortex ring formation. J. Fluid Mech. 360: 121-140

Hama FR (1962) Streaklines in a perturbed shear flow. Phys. Fluid 5: 644-650 
Izumi K, Kuwahara, K (1983) Unsteady flow field, lift and drag measurements of impulsively started elliptic cylinder and circular-arc airfoil. AIAA Paper 83-1711

Lamb H (1932) Hydrodynamics, sixth edn. Cambridge Univ. Press

Linden PF, Turner JS (2004) 'Optimal’ vortex rings and aquatic propulsion mechanisms. Proc.

Roy. Soc. B271: 647-653

Matsuuchi K, Miwa T, Nomura T, Sakakibara J, Shintani H, Ungerechts BE (2009) Unsteady flow field around a human hand and propulsive force in swimming. J. Biomech. 42: 42-47

Milne-Thomson LM (1966) Theoretical aerodynamics. Macmillan, New York

Morikawa K, Grönig H (1995) Formation and structure of vortex systems around a translating and oscillating airfoil. Z. Flugwiss. Weltraumforsch. 19: 391-396

Müller UK, van den Heuvel BLE, Stamhuis EJ, Videler JJ (1997) Fish foot prints: morphology and energetics of the wake behind a continuously swimming mullet (Chelon labrosus Risso). J. Exp. Biol. 200: 2893-2906

Nauen JC, Lauder GV (2002) Hydrodynamics of caudal fin locomotion by chub mackerel, Scomber japonicus (Scombridae). J. Exp. Biol. 205: 1709-1724

Parker K, von Ellenrieder KD, Soria J (2005) Using stereo multigrid DPIV (SMDPIV) measurements to investigate the vortical skelton behind a finite-span flapping wing. Exp. Fluids 39: $281-298$

Parker K, Soria J, von Ellenrieder, KD (2007a) Thrust measurements from a finite-span flapping wing. AIAA J. 45(1): 58-70

Parker K, von Ellenrieder KD, Soria J (2007b) Morphology of the forced oscillatory flow past a finite-span wing at low Reynolds number. J. Fluid Mech. 571: 327-357

Prasad AK, Adrian RJ (1993) Stereoscopic particle image velocimetry applied to liquid flows.

Exp. Fluids 15: 49-60

Prasad AK, Jensen K (1995) Scheimpflug stereocamera for particle image velocimetry in liquid flows. Appl. Opt. 34: 7092-7099

Sakakibara J, Nakagawa M, Yoshida M (2004) Stereo-PIV study of flow around a maneuvering fish. Exp. Fluids 36: 282-293

Sane SP (2003) The aerodynamics of insect flight. J. Exp. Biol. 206: 4191-4208

Sengupta TK, Lim TT, Sajjan SV, Ganesh G, Soria J (2007) Accelerated flow past a symmetrical aerofoil: experiments and computations. J. Fluid Mech. 591: 255-288

Soria J, New TH, Lim TT, Parker K (2003) Multigrid CCDPIV measurements of accelerated flow past an airfoil at an angle of attack of 30 . Exp. Therm. Fluid Sci. 27: 667-676

Taylor GI (1938) The spectrum of turbulence. Proc. Roy. Soc. 164: 476-490

Taylor GK, Nudds RL, Thomas ALR (2003) Flying and swimming animals cruise at a Strouhal number tuned for high power efficiency. Nature 425: 707-711

Triantafyllou MS, Triantafyllou GS, Yue DKP (2000) Hydrodynamics of fishlike swimming. Ann. Rev. Fluid Mech. 32: 33-53

Triantafyllou MS, Techet AH, Hover FS (2004) Review of experimental work in biomimetic foils. IEEE J. Oceanic Eng. 29: 585-594 
TroolinDR, Longmire EK, Lai WT (2006) Time resolved PIV analysis of flow over a NACA 0015 airfoil with Gurney flap. Exp. Fluids 41: 241-254

von Ellenrieder KD, Parker K, Soria J (2003) Flow structures behind a heaving and pitching finitespan wing. J. Fluid Mech. 490: 129-138

Wolfgang MJ, Triantafyllou MS, Yue DKP (1999) Visualization of complex near-body transport process in flexible-body propulsion. J. Visual. 2: 143-151

Fig. 1 Experimental setup for stereo-PIV

Fig. 2 Measurement planes for stereo-PIV

Fig. 3 Shape and material of the wing: $b$ is the span, and $c$ is the chord length of the wing

Fig. 4 Schematic of the pitching motion of the wing and its drive unit

Fig. 5 3D vortex structure in the wing wake: left, $t^{*}=0.45$; right, $t^{*}=0.95$

Fig. 6 3D vortex structure of three components of the vorticity, $\omega_{x}(\mathrm{a}), \omega_{y}(\mathrm{~b})$, and $\omega_{z}(\mathrm{c})$ : left, $t^{*}=$ 0.45 ; right, $t^{*}=0.95$

Fig. 7 Illustration of calculation of circulation $\Gamma$

Fig. 8 Illustration of calculation of area $A_{y z}$

Fig. 9 Circulation $\Gamma$ and acceleration (arbitrary scale) as a function of dimensionless time

Fig. 10 Area $A_{y z}$ as a function of dimensionless time

Fig. 11 Propulsive force $F_{X}$ as a function of dimensionless time

Fig. 12 3D vortex structure reconstructed using 2D data at $t^{*}=0.95$

Fig. 13 Illustration of calculation of the circulation around a vortex ring

Table 1 Experimental conditions for stereo-PIV

Table 2 Circulation around the vortex ring for various pitching amplitudes and cross-sections: center plane and planes of $\theta_{\mathrm{w}}=20^{\circ}(\mathrm{LH})$ and $160^{\circ}(\mathrm{RH})$ 


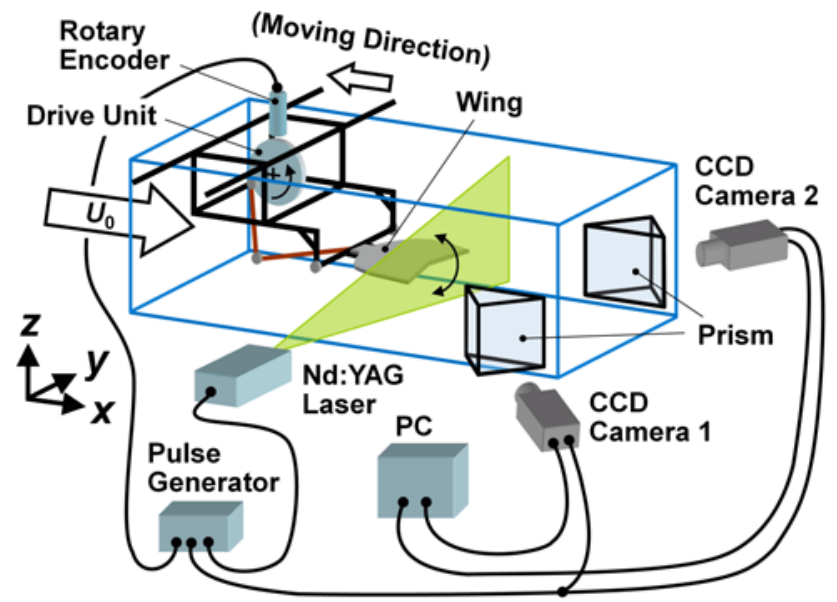

FIG1

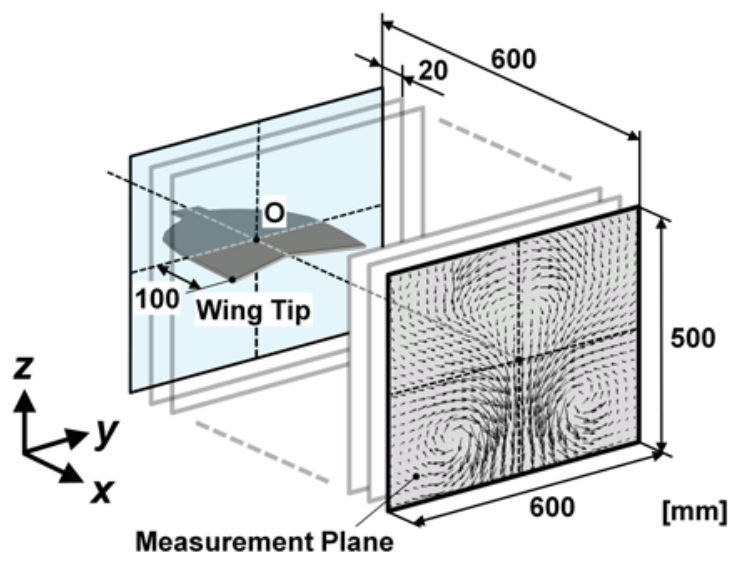

FIG2

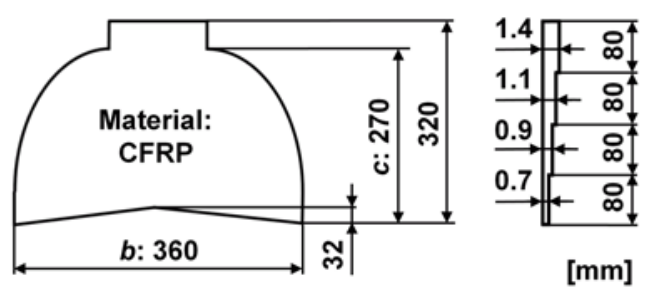

FIG3 


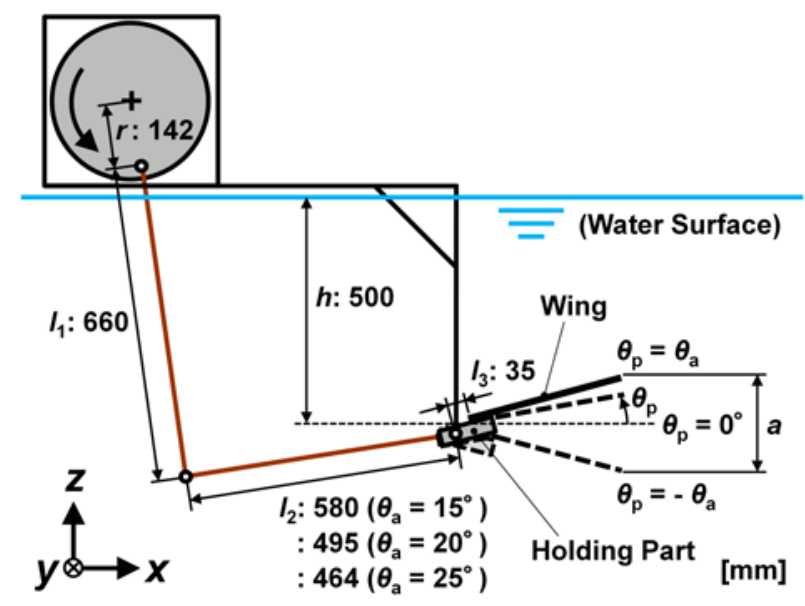

FIG4 

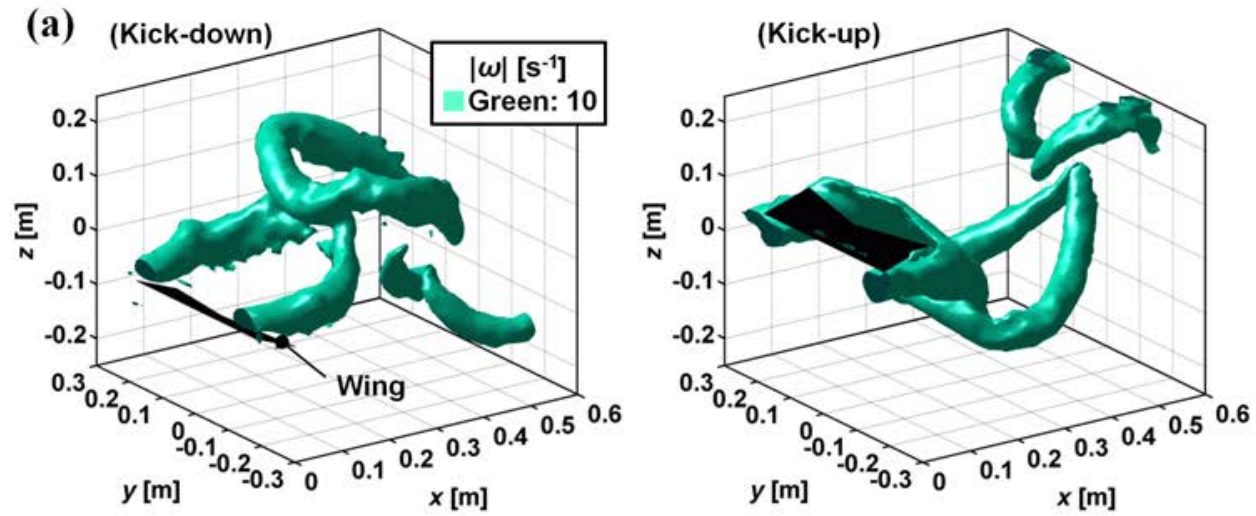

(b) (Kick-down)
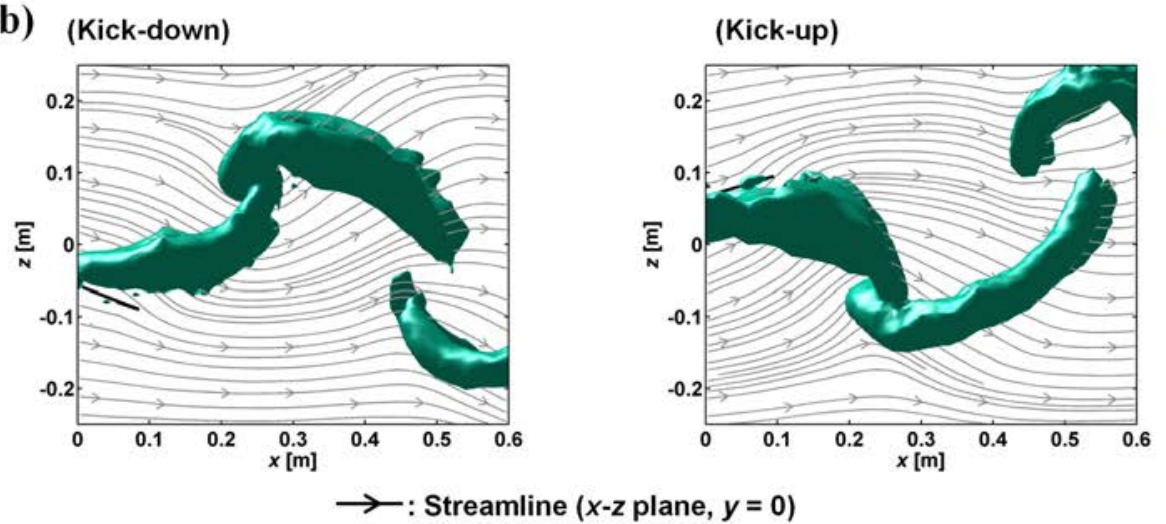

(c) (Kick-down)

(Kick-up)
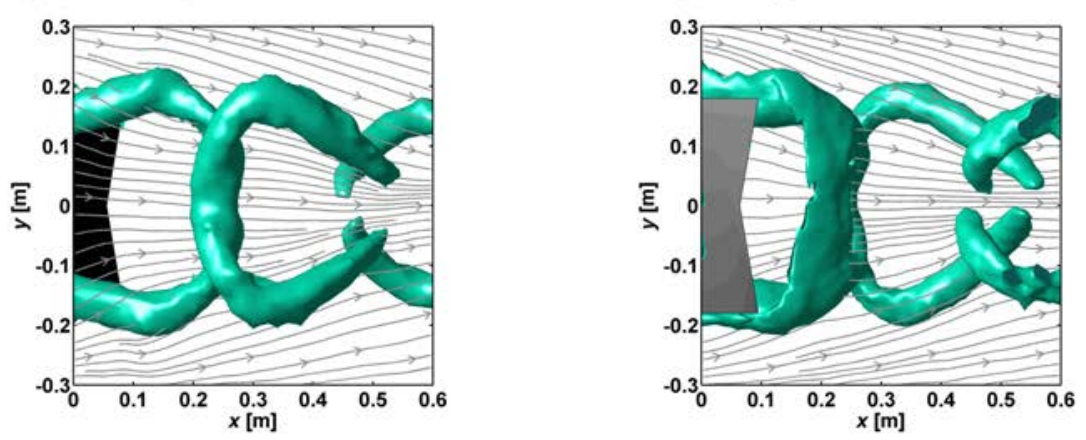

$\longrightarrow$ : Streamline $(x-y$ plane, $z=0)$

FIG5 

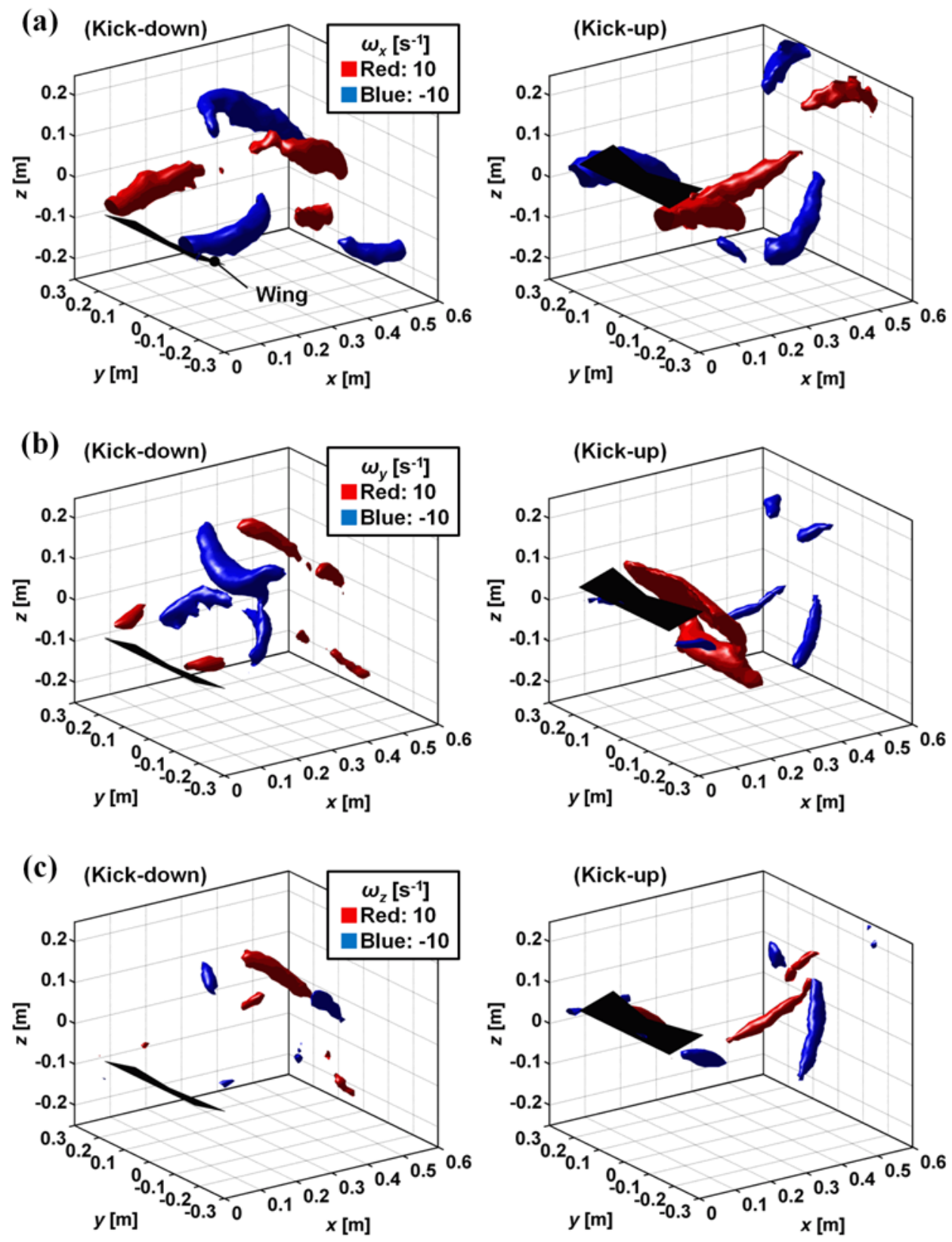

FIG6 


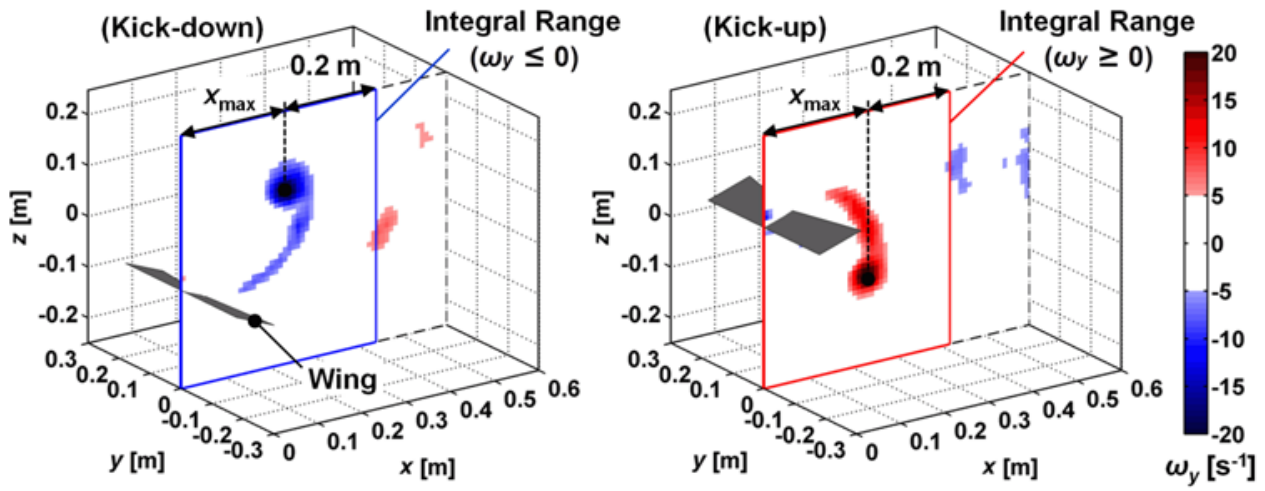

FIG7

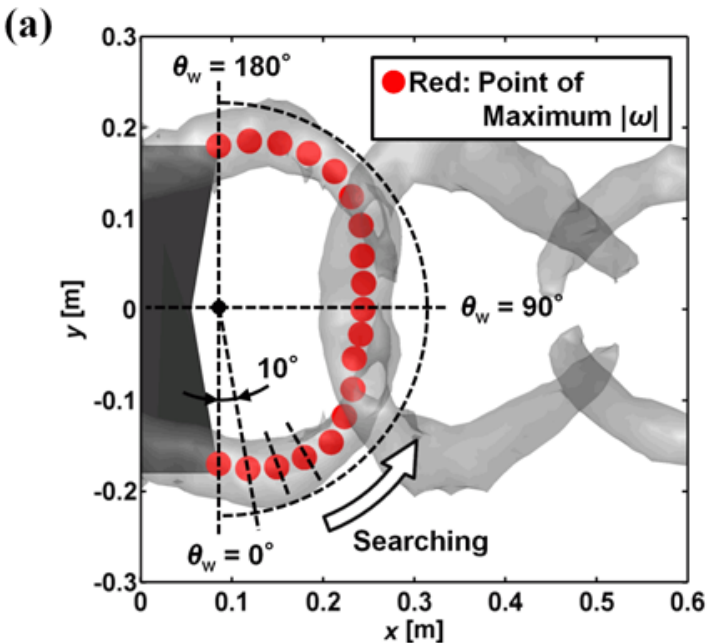

(b)

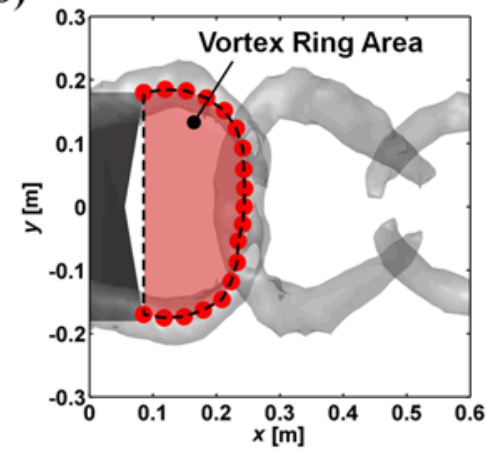

(c)

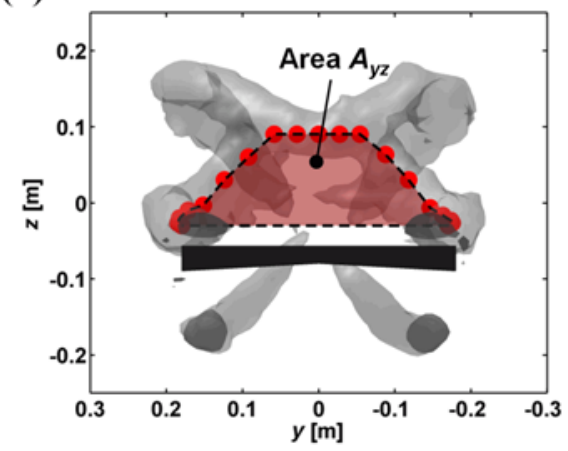

FIG8 


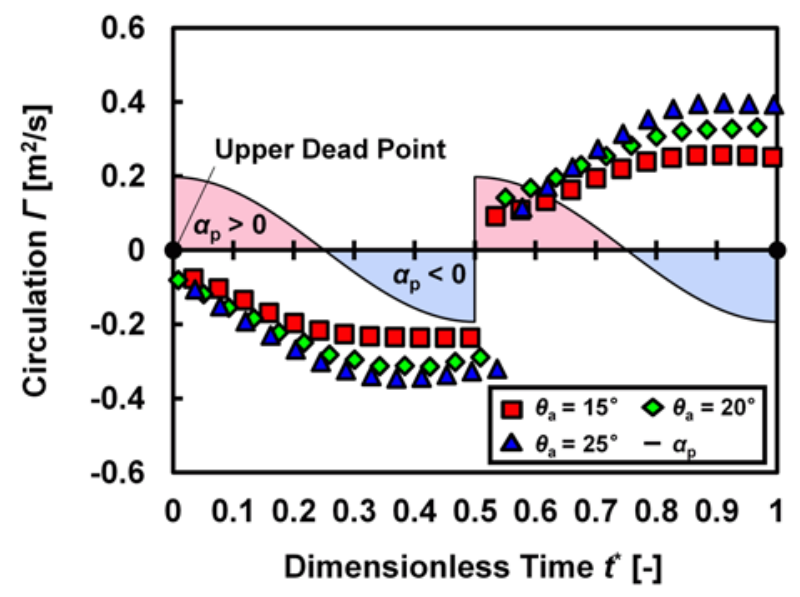

FIG9

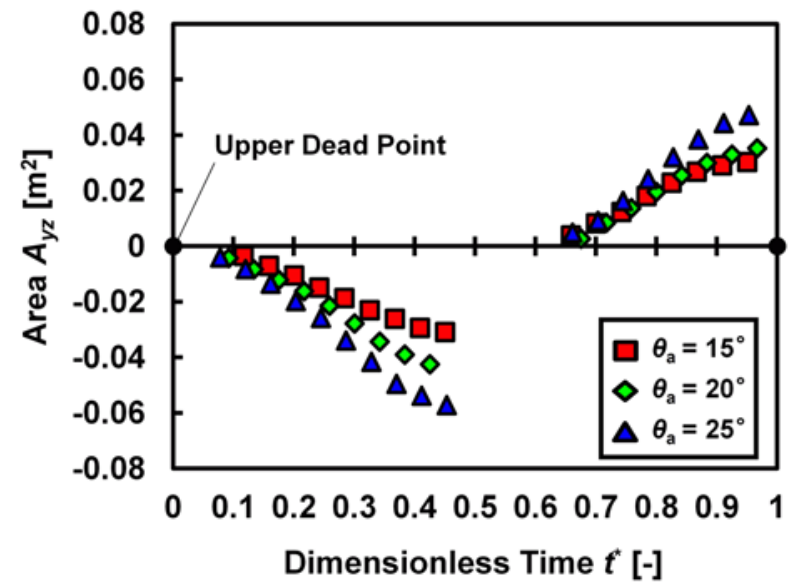

FIG10

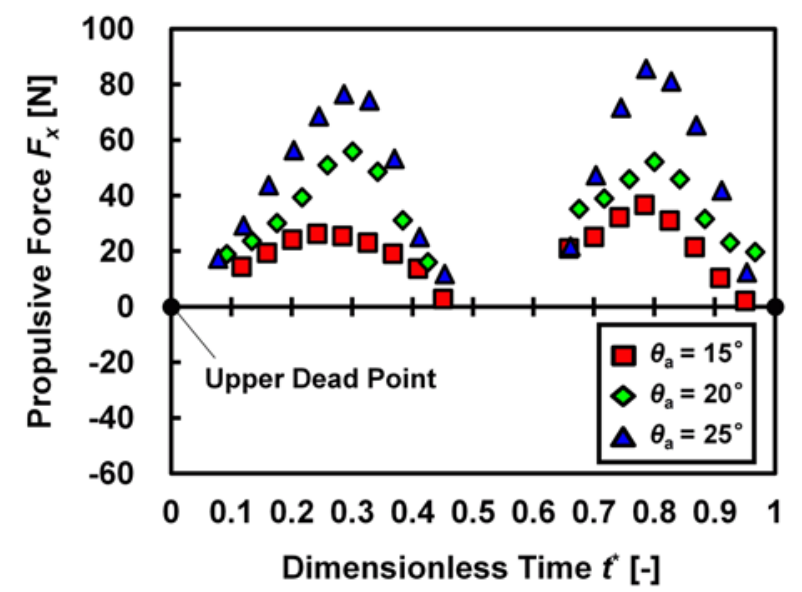

FIG11 


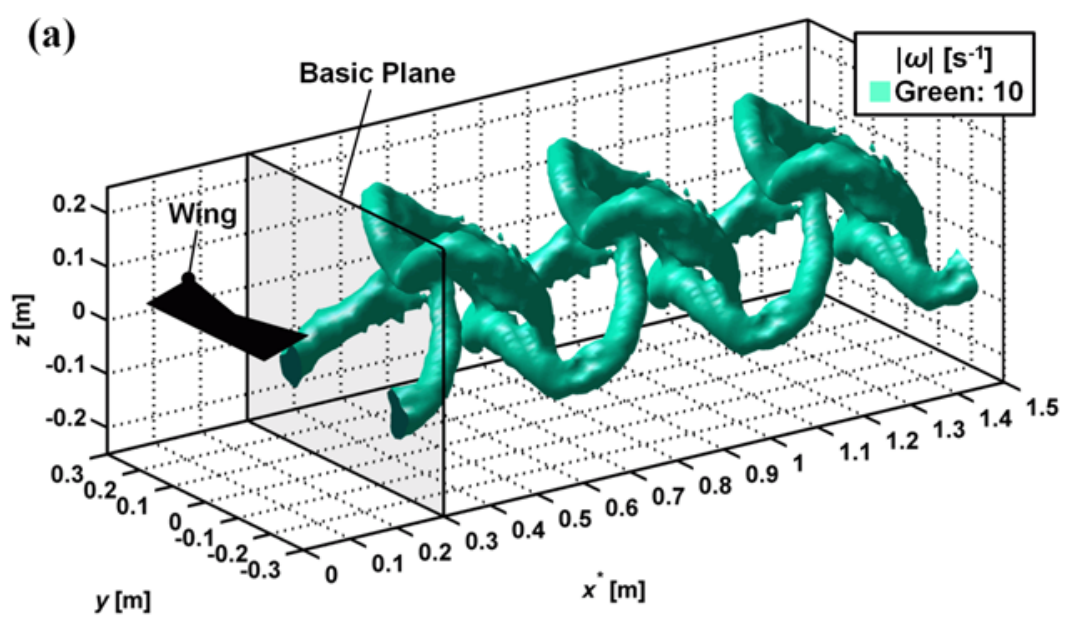

(b)

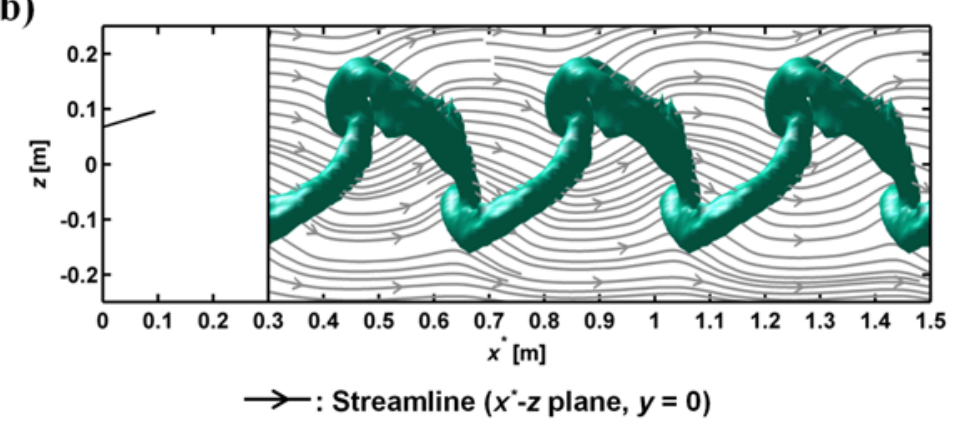

(c)

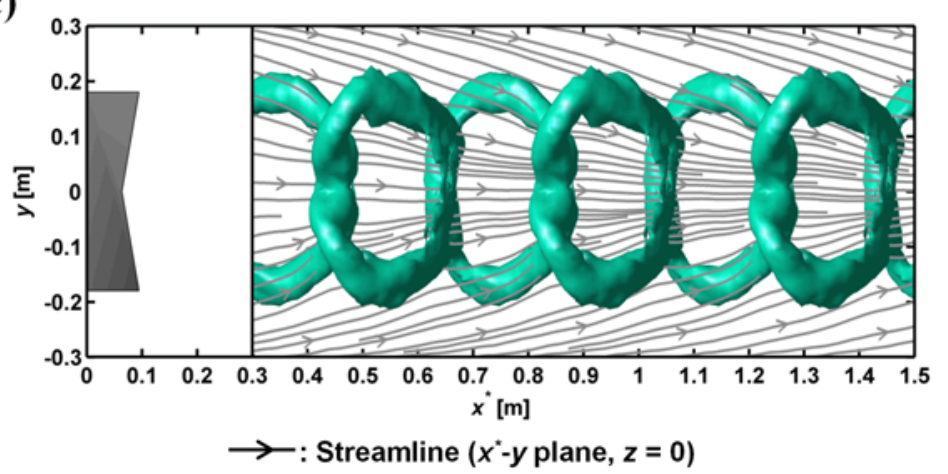

FIG12 
(a)

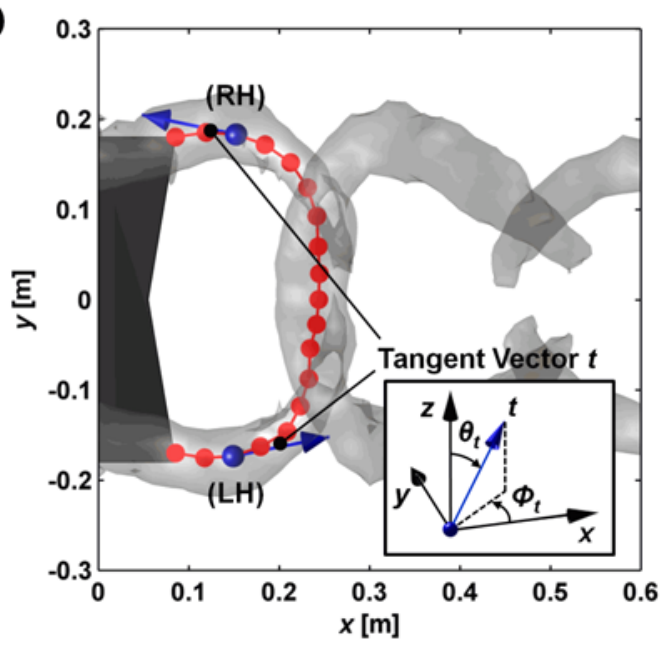

(b)

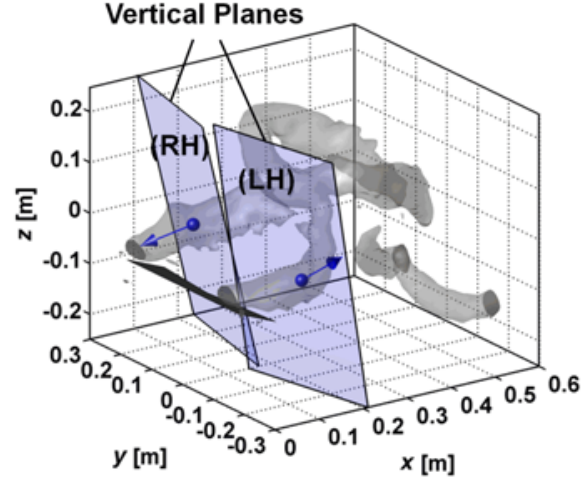

(c) Integral Range

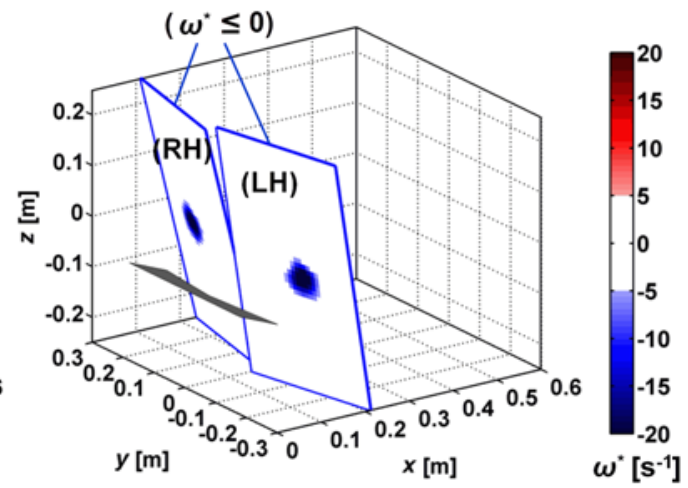

FIG13 


\begin{tabular}{lllll}
\hline$\theta_{\mathrm{a}}[\mathrm{deg}]$. & $a[\mathrm{~m}]$ & $f_{\mathrm{p}}[\mathrm{Hz}]$ & $U_{0}[\mathrm{~m} / \mathrm{s}]$ & St $[-]$ \\
\hline 15 & 0.195 & 1.0 & 0.5 & 0.39 \\
20 & 0.243 & 1.0 & 0.5 & 0.48 \\
25 & 0.292 & 1.0 & 0.5 & 0.58 \\
\hline
\end{tabular}

Table 1 


\begin{tabular}{llllll}
\hline$\theta_{\mathrm{a}}$ [deg.] & Motion & $\begin{array}{l}\Gamma[1 / \mathrm{s}] \\
(\text { Center })\end{array}$ & $\begin{array}{l}\Gamma[1 / \mathrm{s}] \\
(\mathrm{L})\end{array}$ & $\begin{array}{l}\Gamma[1 / \mathrm{s}] \\
(\mathrm{R})\end{array}$ & $(\mathrm{L}+\mathrm{R}) / 2 /$ Center $[\%]$ \\
\hline 15 & Kick-down $\left(t^{*}=0.45\right)$ & -0.24 & -0.14 & -0.13 & 56 \\
& Kick-up $\left(t^{*}=0.95\right)$ & 0.26 & 0.17 & 0.18 & 67 \\
20 & Kick-down $\left(t^{*}=0.43\right)$ & -0.31 & -0.17 & -0.16 & 53 \\
& Kick-up $\left(t^{*}=0.93\right)$ & 0.33 & 0.21 & 0.23 & 67 \\
25 & Kick-down $\left(t^{*}=0.45\right)$ & -0.34 & -0.18 & -0.18 & 53 \\
& Kick-up $\left(t^{*}=0.95\right)$ & 0.40 & 0.25 & 0.26 & 64 \\
\hline & (Shown in Fig. 9)
\end{tabular}

Table 2 